\title{
The use of continuous foetal monitoring technologies that enable mobility in labour for women with complex pregnancies: A survey of Australian and New Zealand hospitals
}

\author{
Deborah Fox $^{\mathrm{a}, *}$, Robyn Maude ${ }^{\mathrm{b}}$, Rebecca Coddington ${ }^{\mathrm{a}}$, Rachael Woodworth ${ }^{\mathrm{a}}$, Vanessa Scarf ${ }^{\mathrm{a}}$, \\ Kylie Watson ${ }^{c}$, Maralyn Foureur ${ }^{\mathrm{d}}$ \\ ${ }^{a}$ Centre for Midwifery, Child and Family Health, University of Technology Sydney, P.O. Box 123, Broadway, NSW 2007 Australia \\ ${ }^{\mathrm{b}}$ Victoria University of Wellington, New Zealand \\ ${ }^{\mathrm{c}}$ University of Manchester, Manchester, United Kingdom \\ d University of Newcastle, NSW, Australia
}

\section{A R T I C L E I N F O}

\section{Keywords:}

Survey

Mobility in labour

Intrapartum care

Labour complications

Foetal wellbeing

\begin{abstract}
A B S T R A C T
Objective: Freedom of movement and choice of positioning in labour and birth is known to enhance physiological processes and positive experiences for women during childbirth. Continuous foetal monitoring technologies that enable mobility in labour for women with complex pregnancies, such as wireless CTG, have been marketed for clinical use in most high resource settings since 2003 but there is a paucity of midwifery literature about its clinical use. The aim of this survey was to determine how often, and for whom, wireless and beltless technologies are being used in maternity settings across Australia and New Zealand and to identify any barriers to their uptake. Design: A survey tool developed by Watson et al. (2018) for use in the United Kingdom was adapted for the Australian/New Zealand context. One Maternity Unit Manager or key midwifery clinician from each of 208 public and private hospitals across Australia and New Zealand was invited by email to participate in an online survey between October 2019 and January 2020. Descriptive statistics were used to describe the characteristics of the facilities and the frequency of availability of the monitors. Free text responses were thematically analysed. Findings: The survey received a high (71\%) response rate from a range of public and private hospitals in urban and rural settings. Women's freedom of movement and sense of choice and control in labour were seen by most respondents to be positively influenced by wireless monitoring technology. Most facilities reported having at least one wireless or beltless foetal monitor available, however, results suggest that many women consenting to continuous monitoring still do not have access to technology that enables freedom of movement.

Keyconclusions: Further research is required to explore the barriers and facilitators to enabling freedom of movement and positioning to all women in childbirth, including those women with complex pregnancies who may consent to continuous foetal monitoring.
\end{abstract}

\section{Introduction}

A variety of methods are used in clinical practice to monitor foetal well-being during childbirth. These include intermittent auscultation of the foetal heart, using either a Pinard fetoscope or handheld Doppler, and technologies that enable the foetal heart and uterine activity to be measured continuously. Intermittent auscultation for healthy women and babies at term is supported by evidence (Alfirevic et al. 2017; Maude, Skinner and Foureur, 2014; NICE 2017) and affords women freedom of movement and choice of position in labour and birth. For women identified as having complex pregnancies or being at high risk of complications in labour, continuous cardiotocography (CTG) is listed in international clinical guidelines as the recommended method of monitoring foetal well-being (FIGO 2015; RANZCOG 2019). This recommendation is regarded by some as contentious, with the results of a recent systematic review and meta-analysis (Small et al. 2019) suggesting that more robust evidence is required to investigate whether continuous monitoring is beneficial.

Guidelines from Australia suggest that more than half of the 310,000 women giving birth each year (AIHW 2018) are recommended to have

\footnotetext{
* Corresponding author.

E-mail addresses: Deborah.Fox@uts.edu.au (D. Fox), Robyn.Maude@vuw.ac.nz (R. Maude), Rebecca.Coddington@uts.edu.au (R. Coddington),

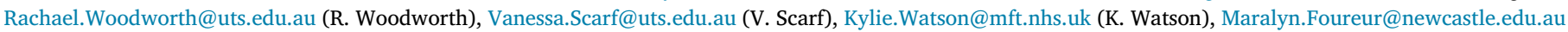
(M. Foureur).
} 
continuous CTG monitoring due to complications, risk factors or interventions such as induction of labour (NSW Health 2016). Similarly, in New Zealand, half of all the women giving birth in 2017 experienced some form of intervention during labour and birth, including induction or augmentation of labour and/or use of epidural analgesia which are indications associated with recommendations for continuous CTG monitoring (New Zealand Ministry of Health 2019). Anecdotal information suggests that in some clinical settings in the region, healthy women with full term pregnancies who are at low risk of complications are often monitored continuously, despite a lack of evidence for this practice.

Traditionally, continuous CTG monitoring requires a woman in labour to wear two tight elastic belts around her abdomen and to be connected by metre length wiring. The purpose of the belts is to hold two transducers in place on the woman's abdomen. This technology restricts a woman's mobility during labour and may limit her choice of position whilst giving birth (Alfirevic et al. 2017). Restricted mobility in labour results in increased rates of intervention including caesarean section (Lawrence et al. 2013; Priddis et al. 2012; WHO 2018; Zang et al. 2020) which carry significant short and long-term implications for women and their babies (Sandall et al. 2018). Restricting a woman's freedom of movement also leads to a lack of choice and control over both her body and her environment (Albers et al. 1997). Choice and control are of high importance to women in labour (Albers et al. 1997; Downe et al. 2018; Hindley et al. 2008; Olza et al. 2018; Priddis et al. 2012). For many women, making informed choices about foetal monitoring forms part of their sense of control (Hindley et al. 2008). Paradoxically, reduced maternal sense of control results in an increased need for pharmacological analgesia such as epidural (Albers et al. 1997), which acts to further reduce women's mobility in labour.

Freedom of movement in labour has been shown to result in shorter labours, increased uterine contractility, increased maternal comfort levels and reduced need for pharmacological pain management (Albers et al. 1997; Lawrence et al. 2013; Priddis et al. 2012). Continuous foetal monitoring technologies that enable mobility in labour, such as wireless CTG, have been marketed for clinical use in most high resource settings since 2003 but there is a paucity of midwifery literature about its clinical use. A recent survey of the use of wireless CTG in the United Kingdom (UK) demonstrated that whilst $62 \%$ of responding hospitals possessed at least one wireless CTG machine, only $18 \%$ had more than three available (Watson et al. 2018). This indicates that the majority of women being continuously monitored are still being attached to the wired technology and that there is a low rate of availability of the wireless technology in the UK (Watson et al. 2018). To date, there is no research published about the availability of wireless foetal monitoring technologies throughout Australia and New Zealand.

This study is a survey of Australian and New Zealand maternity health facilities about their use of continuous foetal monitoring technologies that enable mobility in labour for women with complex pregnancies. The aim of this research is to determine how often, and for whom, wireless and beltless technologies are being used in maternity settings across Australia and New Zealand and to identify any barriers to their uptake. The results will contribute to the design of future research and implementation work aiming to upscale the use of technologies that enable freedom of movement in labour for women with complex pregnancies.

\section{Methods}

For the purpose of this study, a survey tool developed by Watson et al. (2018) for use in the UK was adapted for the Australian/New Zealand context by four members of the research team. Adapting the survey primarily involved changing the language in some of the questions to ensure relevance to the local contexts in both Australia and New Zealand. A copy of the survey questions will be made available by the first author on email request. Ethical approval to conduct the study as an online survey was granted by the University of Technology Human
Research Ethics Committee (HREC ETH19-3334) in Australia and ratified by the Victoria University of Wellington Human Research Ethics Committee in New Zealand. A fifth member of the research team developed the structure and functioning of the online survey in REDCap, a secure web application for building and managing online surveys.

\section{Setting}

Public and private hospitals with greater than 100 births per year from all states and territories of Australia and New Zealand were included. A total of 208 public and private hospitals were invited to participate in the survey; 187 Australian facilities and 21 New Zealand facilities. Out of these 208 hospitals, $78 \%$ were public hospitals and $22 \%$ were private/other hospitals. Sites were excluded if they did not provide any form of continuous electronic foetal monitoring during labour.

\section{Population}

From each hospital, one Maternity Unit Manager and/or key midwifery clinician was invited by email to participate as a representative of the organisation. Ethical approval was granted to use publicly available contact details to contact potential participants. Email addresses were obtained via publicly available hospital websites or by using publicly available telephone numbers to call the hospitals, explain the study and request an email address. Email addresses were then added as participants to REDCap. A unique survey link was created for each participating maternity facility and the survey was distributed via invitation emails to each participant containing this unique link.

\section{Data collection}

As survey responses were de-identified, a unique survey link enabled the researchers to determine which maternity facilities had not yet responded to the survey, so that personalised follow up emails were able to be sent. The unique survey link also enabled the original participant, from each maternity facility, to forward the survey to another midwifery representative from their facility in cases where the original participant was unable or unwilling to represent their maternity facility by participating in the survey. Participants were therefore encouraged to do this in the invitation email and in follow up emails, leading to a lower attrition rate of participants. Having a unique survey link also prevented the survey being completed by multiple participants from the same hospital facility, as once one midwifery representative from the facility responded, the survey would close to future participants. Participants were able to use their unique survey link to re-access any incomplete responses, if they were unable to attend to the survey in one sitting. It was explained to participants in the Participant Information Sheet that informed consent was implied if they completed the online survey through the link provided.

The survey opened on the 21st of October 2019 and remained open for three months until the 19th of January 2020. Four follow up emails were sent during this period, in which participants were encouraged to forward the email on to other midwifery representatives from their maternity facility if they were unable to participate in the survey themselves.

\section{Data analysis}

De-identified quantitative data were transferred from REDCap to a Statistical Package for Social Sciences (SPSS), for the purposes of analysis. Descriptive statistics were used to describe the characteristics of the facilities and the frequency of availability of the monitors. Free text responses were thematically analysed using the methods of Braun and Clarke (2006). Data were collated in a Word document and distributed to three team members for analysis. Each team member individually reviewed all free text responses in order to identify patterns in the data 
Table 1

Characteristics of responding facilities.

\begin{tabular}{|c|c|c|}
\hline & $\mathrm{n}$ & $\%$ \\
\hline \multicolumn{3}{|l|}{ Type of facility } \\
\hline Obstetric led & 109 & 74 \\
\hline Midwifery led & 20 & 14 \\
\hline Other (MW and OB led; GP shared care/MW led) & 19 & 12 \\
\hline \multicolumn{3}{|l|}{ Level of service } \\
\hline Tertiary & 40 & 27 \\
\hline Secondary & 93 & 63 \\
\hline Other* & 15 & 10 \\
\hline \multicolumn{3}{|l|}{ Health sector } \\
\hline Public hospital & 120 & 81 \\
\hline Private/other hospital & 28 & 19 \\
\hline \multicolumn{3}{|l|}{ Remoteness } \\
\hline Metropolitan & 71 & 48 \\
\hline Rural/remote & 65 & 44 \\
\hline Other*** & 12 & 8 \\
\hline \multicolumn{3}{|l|}{ State } \\
\hline NSW & 51 & 35 \\
\hline QLD & 24 & 16 \\
\hline Vic & 24 & 16 \\
\hline WA & 14 & 10 \\
\hline SA & 10 & 7 \\
\hline NT & 5 & 3 \\
\hline ACT & 2 & 1 \\
\hline Tas & 2 & 1 \\
\hline $\mathrm{NZ}$ & 16 & 11 \\
\hline \multicolumn{3}{|l|}{ Midwifery role } \\
\hline Midwifery Unit Manager & 77 & 52 \\
\hline CMC/CMS/CME & 41 & 28 \\
\hline Clinical Midwife & 17 & 12 \\
\hline DONM/ Clinical Director & 10 & 7 \\
\hline Other senior midwife & 3 & 2 \\
\hline
\end{tabular}

CMC: Clinical Midwifery Consultant, CMS: Clinical Midwifery Specialist, CME: Clinical Midwifery Educator, DOMN: Director of Nursing and Midwifery.

* Regional facility/ Level 3 Maternity service capability level 2 Nursery/ Regional/rural unit/Primary low risk unit

** regional facility/urban NZ also have primary midwifery led/

both urban and rural and risk primary 40 mins from tertiary unit

and develop initial codes. Codes were derived directly from the data and the research team then met to compare, contrast, discuss and develop the data into agreed codes and early themes. Themes were discussed and further refined as the qualitative findings were written up.

\section{Results}

\section{Response rate}

The survey achieved a 71\% response rate, with 148/208 maternity facilities responding. From each facility, one Maternity Unit Manager and/or key midwifery clinician was invited to represent their organisation. In the Findings, respondents may be referred to as either the 'maternity facility' or the 'midwife respondent', depending upon the context.

Table 1 depicts the characteristics of the responding facilities, including their funding source, general location (state/territory, ru$\mathrm{ral} /$ metropolitan), predominant model of care and role of the responder. Respondents comprised $81 \%$ public hospitals and $19 \%$ private/other hospitals, a close representation of the initial sample, which was made up of $78 \%$ public hospitals and $22 \%$ private/other hospitals. Of 187 Australian facilities contacted, 133 responded (71\%). Similarly, 16 out of the $21(71 \%)$ New Zealand facilities responded to the survey. A total of $74 \%$ of responding hospitals identified as obstetric led facilities $(n=109)$, $14 \%$ identified as midwifery led facilities $(n=20)$ and $13 \%$ identified as 'other' ( $n=19)$. Of the 19 facilities that identified as 'other', $79 \%$ stated that they had both obstetric and midwifery led models of care in their facility $(n=15)$ and $21 \%$ indicated that their facility offered shared care
Table 2

Number of wireless/beltless monitors by births per year.

\begin{tabular}{lll}
\hline Number of births & Number of hospitals & Range of number of monitors (Mean) \\
\hline $100-500$ & 44 & $1-5(1.8)$ \\
$501-1000$ & 20 & $1-6(2.9)$ \\
$1001-2000$ & 30 & $1-12(4.0)$ \\
$2001-3000$ & 11 & $2-15(7.2)$ \\
$3001-4000$ & 8 & $1-17(9.4)$ \\
$4001-5000$ & 3 & $9-16(12.3)$ \\
$>5000$ & 10 & $1-20(8.5)$ \\
Total: & $126^{*}$ &
\end{tabular}

* Missing data $\mathrm{n}=2$

between midwives and either a General Practitioner (GP) or Obstetrician $(n=4)$.

The number of annual births per year for the 148 responding maternity facilities ranged from 100 births per year to 10000 births per year. The distribution of births per year for the 148 responding maternity facilities was positively skewed, with a median of 1150 births per year and interquartile range (IQR) of 1901 births per year (See Fig. 1).

\section{Findings}

Characteristics of maternity facilities that had wireless/beltless monitoring available

Of the 148 maternity facilities that responded to the survey, 128 $(87 \%)$ stated that they had at least one wireless/beltless continuous foetal monitor available. Annual birth rates at these facilities ranged from 100 births per year to 10000 births per year with a median of 1025 births per year (IQR $=1848$ births per year). Twenty maternity facilities responded that they did not have any wireless/beltless monitoring available. Annual birth rates at these facilities ranged from 150 births per year to 5700 births per year with a median of 1400 births per year (IQR $=1938$ births per year).

Wireless/beltless monitoring was available in $88 \%$ of maternity units that identified as public $(n=105)$ and $82 \%$ that identified as Private/other $(n=23)$. Wireless/beltless monitoring was available in $87 \%$ of maternity units that identified as obstetric led $(n=95), 85 \%$ that identified as midwifery led $(n=17)$ and $84 \%$ that identified as 'other' $(n=16)$. Wireless/beltless monitoring was available in $82 \%$ of maternity units that identified as metropolitan $(n=58), 94 \%$ of maternity units that identified as rural/remote $(n=61)$ and $75 \%$ that identified as 'other' $(n=9)$.

\section{Availability and accessibility}

Of the 128 facilities that stated they had wireless/beltless CTG monitors, most facilities reported having only a few machines available (see Table 2). Almost half had one or two machines, including facilities that had over 5000 births per year. In fact, of the ten hospitals with a 500110,000 yearly birth-rate, five hospitals have less than five wireless monitors. Only eleven facilities stated that they had more than 10 machines available.

When asked how long wireless/beltless monitoring had been available in their maternity facilities, $60 \%$ stated it had been available for $0-5$ years $(n=67), 33 \%$ stated it had been available for $>5$ to 10 years $(\mathrm{n}=37), 5 \%$ stated it had been available for $>10$ to 15 years $(\mathrm{n}=5)$ and $2 \%$ stated it had been available for over 15 years $(n=2)$. Seventeen respondents either did not provide a response to this question or provided unclear responses such as 'several years'.

In order to gain a sense of the level of accessibility of the machines to women and midwives, we asked the maternity facilities where they store their wireless/beltless monitors. Almost half stated that a machine was placed in every birthing room, suggesting that all women consenting to continuous monitoring in labour in those facilities have the opportunity to use wireless/beltless technology. A fifth of the respondents stated they had machine/s stored in only some birthing rooms and a similar number 


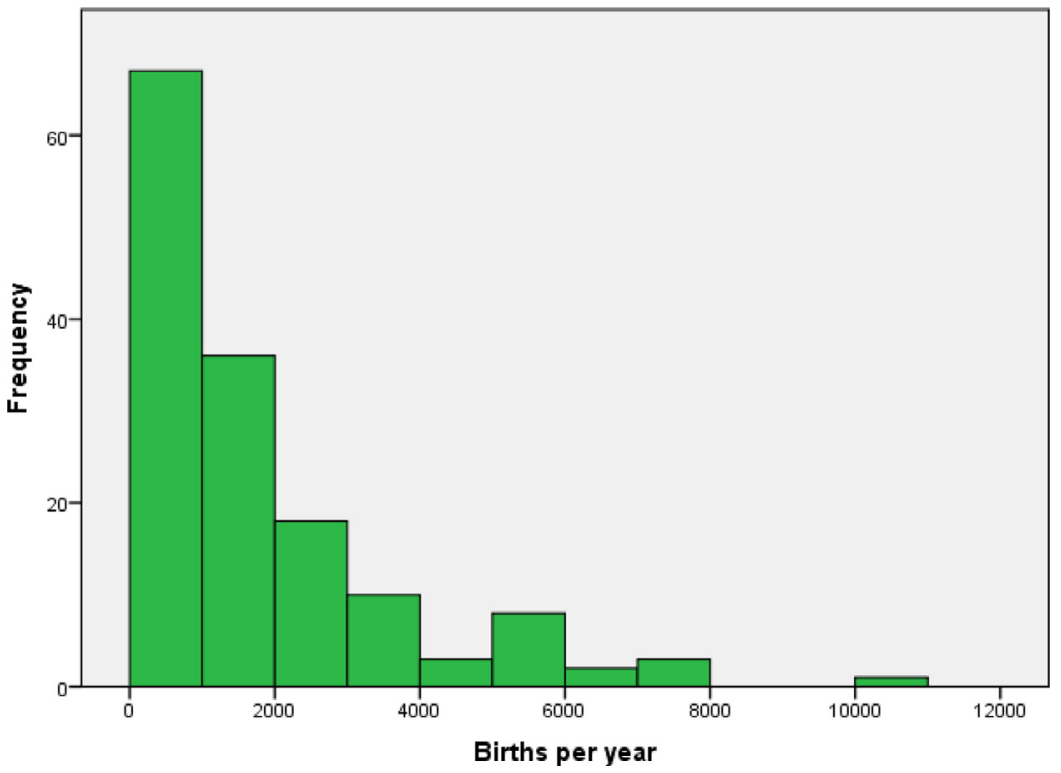

Fig. 1. Distribution of births per hospital per year.
Table 3

Places where wireless/beltless machines are stored.

\begin{tabular}{ll}
\hline Place where machine/s is stored & N (\%) \\
\hline Machine/s remain in every room & $56(44.8)$ \\
Machine/s remain in only some rooms & $26(20.8)$ \\
Machine/s kept in a hallway or storeroom & $22(17.6)$ \\
Other & $21(16.8)$ \\
Total* & $125(100)$ \\
\hline
\end{tabular}

* Missing data $\mathrm{n}=3$

kept the machine/s stored in a hallway or storeroom (see Table 3). Other responses included answers such as 'Combination of all of the above', 'Kept in birth suites only', 'Kept in one room but moved to whichever birth suite it is needed in' and 'Swapped from room to room as needed'.

To gain insight into how often women are being provided with access to wireless/beltless monitoring, participants were asked what percentage of women who use continuous foetal monitoring at their facility can use wireless/beltless monitoring. Forty-three percent $(n=54)$ of participants stated that while wireless or beltless monitoring was available at their facility, it was used by less than half of the women that require continuous foetal monitoring (see Table 4). This indicates that despite a large percentage (87\%) of Australian and New Zealand hospitals having wireless/beltless monitoring available, there is still a large proportion of women unable to access these machines.

Of the 20 maternity facilities (14\%) that responded that they did not have wireless/beltless monitoring available, two-thirds planned to purchase it in the future $(n=13,65 \%)$. When these 13 facilities were asked when they were planning to purchase wireless/beltless machine/s; the majority $(n=11,85 \%)$ responded that they planned to purchase one or more machines within the next 2 years. Only one of those maternity facilities stated that they were planning to purchase a wireless/beltless unit for every birth room in their facility.

\section{Beliefs about the influence of wireless/beltless monitoring upon outcomes during labour and birth}

Facilities with wireless/beltless monitoring available were asked what labour outcomes they believe are positively influenced by the technology. Almost all maternity facilities (98\%) responded to this question and midwife respondents were able to select multiple answers. Most identified freedom of movement ( $n=124,99 \%)$ and women's sense of choice and control $(n=108,86 \%)$ to be positively impacted by wireless/beltless monitoring. Other common responses included normal vaginal birth rate $(n=47,38 \%)$, reduction in epidurals $(n=38,30 \%)$ and reduced length of labour $(n=29,23 \%)$.

\section{Problems encountered when using wireless/ beltless monitoring during labour}

Facilities that have wireless/beltless monitoring available were asked what problems, if any, the midwives encountered when using wireless/beltless monitoring for women in labour. The two most common problems that were identified were loss of contact with the foetal heart rate and/or uterine activity, particularly during water immersion. In addition, frustrations around misplacement of transducers when they were accidentally discarded with bed linen and the high costs associated with replacing them were evident. Other issues that were identified included problems relating to battery life and recharging, damage to transducers and difficulty when monitoring women with increased body mass index (BMI).

\section{Characteristics of women who most often use wireless/beltless monitoring during labour}

Respondents from the maternity facilities that have wireless/beltless monitoring available were also asked for whom wireless or beltless monitoring is most often used. 125 out of the 128 maternity facilities provided a free text response to this question. Responses indicated that wireless monitoring was most commonly used by women without epidurals who wished to be mobile in labour. This included women having an induction of labour and women having a vaginal birth after caesarean (VBAC).

\section{Free text responses}

On completion of the survey, all midwife respondents were asked if they would like to add any free text comments about the use of wireless/beltless technologies for women who require continuous foetal monitoring during labour. Seventy-nine out of the 148 respondents (53\%) provided comments. The three themes that emerged from these comments were, 'Wireless for all women', 'Loss of contact' and 'Anticipating new technology'.

\section{Wireless for all women}

Free text responses were mostly in relation to wireless CTG monitoring. Several midwife respondents indicated that they felt wireless 
Table 4

Percentage of women requiring continuous electronic foetal monitoring who are able to access wireless/beltless machines in labour.

\begin{tabular}{ll}
\hline Percentage of women with access to wireless/beltless monitoring in labour & $\mathrm{N}(\%)$ \\
\hline$<10 \%$ & $13(10.3)$ \\
$10 \%$ to $<30 \%$ & $21(16.7)$ \\
$30 \%$ to $<50 \%$ & $20(15.9)$ \\
$50 \%$ to $<70 \%$ & $23(18.3)$ \\
$70 \%$ or more & $49(38.9)$ \\
Total ${ }^{*}$ & $126(100)$
\end{tabular}

Missing data $\mathrm{n}=2$.

CTG monitoring was beneficial for women and expressed a desire for the wireless technology to be more readily available:

'I believe this is beneficial for all women to use in labour and believe it should be used in our unit'

Midwife respondents who had used both wired and wireless CTG wanted to see an increase in the availability and use of wireless monitoring:

'I wish all our monitors were wireless'

'If CTG monitoring is required, it would be wonderful if we could get all women on wireless CTGs'

'I am happy to use wireless CTG with all women if possible. Some women are very disappointed if this is not available to them [when all] the wireless machines are in use'

In addition to benefits for women, it was recognised that wireless CTG had benefits for midwives. For example, it was seen to reduce potential workplace hazards:

'I think wireless CTG machines are great. Better for WHS [workplace health and safety], as not trying to manoeuvre around lots of wires, especially when likely connected to a drip with multiple lines [during] induction of labour, for example'

\section{Loss of contact}

The second theme that emerged from the free text responses was 'Loss of contact'. Whilst midwife respondents liked the fact that wireless CTG technology provides freedom of movement for women, they reported frequent problems with maintaining contact with the foetal heart rate and uterine activity signals, particularly when women were mobilising in labour:

'[Wireless CTG] is a good tool however it has its drawbacks particularly in the shower and the bath, all fours position, pelvic tilts etc, due to repeated loss of contact'

When loss of contact persisted, midwives reported that women were often asked to move into a position that would allow for clearer auscultation of the foetal heart rate, thus disturbing her labour:

'...These women are often still required to adopt a position that they don't naturally want to get in to, for the sake of monitoring'

Although supportive of women's needs to mobilise in labour, midwives were frustrated by the need to frequently readjust the CTG when loss of contact occurred.

\section{Anticipating new technology}

There was a strong sense that an improved product was required, in order to meet the needs of childbearing women in labour. It was anticipated that a new beltless product would relieve women of the discomfort they experienced when wearing elastic belts to hold the CTG in place:
'I would love to see the new...beltless monitors across [the state] as I find, even though the women love the freedom of no wires, they soon get sick of the tight belts around their bellies'

It was also anticipated that new beltless foetal monitoring technology would reduce the need for midwives to reposition the transducers:

'Alternative methods such as the [beltless] patch system reduce this [need to readjust] dramatically and should be introduced to the Australian market'

Midwife respondents were hopeful that new foetal monitoring technology would limit disruption to the labouring woman by reducing the need to reposition the monitoring device:

'Better technology that reduces the amount of 'fiddling' that midwives need to do to maintain contact would be great!'

\section{Discussion}

This study is the first survey exploring the use of wireless and beltless continuous foetal monitoring in Australian and New Zealand maternity units. Responses were received from maternity facilities in every Australian state and territory as well as a geographically varied spread of maternity facilities from New Zealand. The $71 \%$ response rate compared favourably with the $62 \%$ response rate achieved in the survey from the United Kingdom (UK) (Watson et al. 2018), from which the survey used in this study was adapted. The overall findings of the two studies are aligned, both adding to the paucity of international evidence on enabling freedom of movement and positioning for women who consent to continuous foetal monitoring.

The recognition by midwives that freedom of movement and greater sense of choice and control during childbirth is afforded by wireless/beltless monitoring is a significant finding. Freedom of movement in labour has been shown to result in positive outcomes and experiences for women, including an increase in uterine activity, shorter labours, greater comfort levels and less need for pharmacological pain management (Albers et al. 1997; Lawrence et al. 2013; Priddis et al. 2012). Reduced use of pharmacological pain management in women with complex pregnancies may benefit both maternal and neonatal outcomes. Feelings of choice and control are known to be important to women (Albers et al. 1997; Downe et al. 2018; Hindley et al. 2008; Olza et al. 2018; Priddis et al. 2012). Women with complex pregnancies who consent to continuous monitoring are entitled to benefit from the biological processes that are optimised as a result of being able to mobilise (Kennedy et al. 2018) and to retain their bodily autonomy whilst giving birth.

Whilst acknowledging some imperfections in the currently available wireless/beltless technology, most midwives said that they prefer to use it over the wired technology. The primary reason was because it was seen as a facilitator of freedom of movement and positioning, enhancing women's autonomy during labour and birth. Overall, the challenges associated with using wireless/beltless monitoring demonstrated in this Australia/New Zealand survey were similar to those found in the UK 
(Watson et al. 2018). The main problems identified included frequent misplacement of transducers, loss of contact of foetal heart and/or uterine activity, issues with battery life and recharging, as well as difficulties monitoring women with a high BMI.

This survey highlighted that, although at least one wireless and/or beltless machine was available in most Australian and New Zealand facilities (87\%), access to wireless/beltless technologies for women using continuous monitoring remains limited. Almost half (47\%) of the maternity facilities in Australia and New Zealand have only one or two wireless/beltless machines available and $43 \%$ of maternity facilities provided wireless/beltless technologies to less than half of the women using continuous foetal monitoring. This demonstrates that a large proportion of Australian and New Zealand women continue to be offered only the more restrictive, wired technology. A similar lack of availability of wireless machines was depicted in the survey based in the UK by Watson et al. (2018), which found while $63 \%$ of facilities had at least one wireless CTG machine available, only $18 \%$ of facilities had more than three machines available.

Whilst this study has provided some valuable insights into some of the benefits, disadvantages, barriers and facilitators of wireless/beltless monitoring within Australian and New Zealand facilities, a limitation is that it was unable to provide an in-depth exploration on these concepts. Similar to the UK survey by Watson et al. (2018), it was designed to gain a broad, overall picture of the availability and use of wireless/beltless monitoring. Further qualitative research is planned to provide more indepth data that will elicit a deeper understanding of the subject matter and assist the design of future implementation studies.

Future implementation work aims to increase the use of intermittent auscultation and, for women who consent to continuous monitoring, improve access to wireless/beltless technologies in Australian and New Zealand facilities. An analysis of whether clinical guidelines about foetal monitoring are presented in a manner that encourages practitioners to facilitate freedom of movement and positioning for women being continuously monitored is warranted. Furthermore, there is the potential to replicate and/or adapt this survey in order to gain insight into the availability and use of wireless/beltless monitoring in other countries. Further information about the survey tool may be obtained from the authors.

\section{Conclusion}

The majority (86\%) of Australian and New Zealand maternity facilities have at least one wireless/beltless CTG machine available. Despite this, due to many hospitals only having a small number of machines available, women are often still being required to use traditional, wired cardiotocography. Almost two-thirds (65\%) of the Australian and New Zealand facilities who did not have wireless/beltless machines available, planned to purchase such technology in the future, which indicates its appeal. Almost all the midwives who responded to this survey $(99 \%)$ identified that using wireless/beltless monitoring improved women's freedom of movement in labour. Routine intermittent auscultation, with access to wireless/beltless monitoring technology when required for women with complexities, would provide all women with universal freedom of movement and greater sense of choice and control during their labour and birth experience.

\section{Credit author statement}

Deborah Fox, methodology, gaining ethics approvals, resources, formal analysis, data curation, writing, reviewing and editing, project administration, funding acquisition; Robyn Maude, methodology, gaining ethics approvals, resources, writing, reviewing and editing; Rebecca Coddington, methodology, gaining ethics approvals, resources, formal analysis, data curation, writing, reviewing and editing; Rachael Woodworth, software, resources, formal analysis, data curation, writing, reviewing and editing; Vanessa Scarf, formal analysis, data cura- tion, writing, reviewing and editing; Kylie Watson, conceptualisation, methodology, reviewing and editing; Maralyn Foureur, methodology, reviewing and editing.

\section{Ethical approvals}

Ethics approval to conduct this study was granted by the University of Technology Sydney University Human Research Ethics Committee (HREC ETH19-3334) in Australia and ratified by the Victoria University of Wellington Human Research Ethics Committee in New Zealand.

\section{Funding sources}

Grant funding was provided for this Investigator-led study, to the University of Technology Sydney (UTS), by Philips Medizin Systeme Boeblingen $\mathrm{GmbH}$, Germany. The funder had no role in the study design, data collection, data analysis, interpretation of data, report writing, nor any influence in the decision to submit the article for publication.

\section{Declaration of Competing Interest}

The first author, Dr Deborah Fox, has received honoraria for presenting workshops to clinical midwives on freedom of movement and positioning in labour and birth, from Philips Australia and New Zealand.

\section{Supplementary materials}

Supplementary material associated with this article can be found, in the online version, at doi:10.1016/j.midw.2020.102887.

\section{References}

Albers, L.L., Anderson, D., Cragin, L., Daniels, S.M., Hunter, C., Sedler, K.D., Teaf, D., 1997. The relationship of ambulation in labour to operative delivery. J. Nurse Midwifery 42 (1), 4-8.

Alfirevic, Z., Gyte, G.M.L., Cuthbert, A., Devane, D., 2017. Continuous cardiotography (CTG) as a form of electronic fetal monitoring (EFM) for fetal assessment during labour. Cochrane Database Systematic Rev. (2) doi:10.1002/14651858.CD006066.pub3, Art no. CD006066.

Australian Institute of Health and Welfare, 2018. Australia's mothers and babies 2016 Perinatal statistics series no. 34. Cat. No. PER 97.

Braun, V., Clarke, V., 2006. Using thematic analysis in psychology. Qual. Res. Psychol. 3 (2), 77-101. doi:10.1191/1478088706qp063oa.

Downe, S., Finlayson, K., Oladapo, O., Bonet, M., Gülmezoglu, A.M., 2018. What matters to women during childbirth: a systematic qualitative review. PLoS One 13 (4), e0194906.

Hindley, C., Hinsliff, S.W., Thomson, A.M., 2008. Pregnant women's views about choice of intrapartum monitoring of the fetal heart rate: a questionnaire survey. Int. J. Nurs. Stud. 45, 224-231.

International Federation of Gynecology and Obstetrics (FIGO), 2015. FIGO consensus guidelines on intrapartum fetal monitoring.

Kennedy, H.P., Cheyney, M., Dahlen, H.G., Downe, S., Foureur, M.J., Homer, C.S.E., Jefford, E., McFadden, A., Michel-Schuldt, M., Sandall, J., ... Renfrew, M.J., 2018. Asking different questions: a call to action for research to improve the quality of care for every woman, every child. Birth 45 (3), 222-231. doi:10.1111/birt.12361.

Lawrence, A., Lewis, L., Hofmeyr, G., Styles, C., 2013. Maternal positions and mobility during first stage labour. Cochrane Database Systematic Rev. (10) doi:10.1002/14651858.CD003934.pub4, ArtNo.: CD003934.

Maude, R.M., Skinner, J.P., Foureur, M.J., 2014. Intelligent structured intermittent auscultation (ISIA): Evaluation of a decision-making framework for fetal heart monitoring of low-risk women. BMC Preg. Childbirth 14 (1), 184. doi:10.1186/1471-2393-14-184.

Ministry of Health New Zealand, 2019. Report on Maternity 2017.

National Institute for Health and Care Excellence (NICE), 2017. Intrapartum care for healthy women and babies. NICE Clin. Guideline [CG190].

New South Wales (NSW) Health, Office of Kids and Families, 2016. Maternity - Fetal Heart Rate Monitoring Guideline [GL2016_001]. NSW Health, Sydney, Australia.

Olza, I., Leahy-Warren, P., Benyamini, Y., Kazmierczak, M., Karlsdottir, S.I., Spyridou, A., Crespo-Mirasol, E., Takacs, L., Hall, P.J., Murphy, M., Jondottir, S.S., Nieuwenhuijz, M.J., 2018. Women's psychological experiences of physiological childbirth: a meta-synthesis. BMJ Open, 8, e020347 doi:10.1136/bmjopen-2017-020347.

Priddis, H., Dahlen, H., Schmied, V., 2012. What are the facilitators, inhibitors, and implications of birth positioning? a review of the literature. Women Birth 25 (3), 100-106.

Sandall, J., Tribe, R.M., Avery, L., Mola, G., Visser, G.H., Homer, C.S.E., Gibbons, D., Kelly, N.M., Kennedy, H.P., Kidanto, H., Taylor, P., Temmermann, M., 2018. Short term and long term effects of caesarean section on the health of women and children. Lancet North Am. Ed. 392 (10155), 1349-1357.

Small, K.A., Sidebotham, M., Fenwick, J., Gamble, J., 2019. Intrapartum cardiotocograph monitoring and perinatal outcomes for women at risk: literature review. Women Birth in press. 
The Royal Australian and New Zealand College of Obstetricians and Gynaecologists (RANZCOG), 2019. Intrapartum Fetal Surveillance Clinical Guideline, 4th Edition RANZCOG.

Watson, K., Mills, T., Lavender, T., 2018. The use of telemetry in labour: Results of a national online survey of UK maternity units. Br. J. Midwifery 26 (1), 14-19.
World Health Organization (WHO), 2018. WHO recommendations: Intrapartum care For a Positive Childbirth Experience. World Health Organization, Geneva.

Zang, Y., Lu, Hong., Zhang, H., Huang, J., Zhao, Y., Ren, L., 2020, . Benefits and risks of upright positions during the second stage of labour: An overview of systematic reviews. International Journal of Nursing Studies In Press doi:10.1016/j.ijnurstu.2020.103812. 